\title{
Rhythmic gain control during supramodal integration of approximate number
}

Bernhard Spitzer ${ }^{1}$, Felix Blankenburg ${ }^{1}$, Christopher Summerfield $^{2}$

${ }^{1}$ Neurocomputation and Neuroimaging Unit, Department of Education and Psychology, Freie Universität Berlin, Habelschwerdter Allee 45, 14195 Berlin, Germany

${ }^{2}$ Department of Experimental Psychology, University of Oxford, Oxford OX1 3UD, UK

Corresponding author: Bernhard Spitzer (bernhard.spitzer@fu-berlin.de), Department of Education and Psychology, Freie Universität Berlin, Habelschwerdter Allee 45, 14195 Berlin, Germany, Phone: +49 (0) 30838 55738, Fax: +49 (0) 30838 55693 


\begin{abstract}
According to one view, neural oscillations structure information processing in time, determining whether sensory inputs have a strong or weak impact on behaviour. Recent work showed that during sequential integration of visual inputs, stimuli that fall in the preferred phase of slow (1-3 Hz), endogenous EEG activity carry greater weight in subsequent judgment. Here, we asked two questions. Firstly, is this phenomenon modality-specific, or is it supramodal? Secondly, does this effect occur at the level of sequential encoding, or only during decision formation? We analysed scalp EEG recordings from healthy human participants whilst they compared the approximate number of visual, auditory or somatosensory pulses in two successive intervals (N1 and N2). Despite differences in activity evoked in different domains, a common, slowlyoscillating $(\sim 3 \mathrm{~Hz})$ choice-predictive signal was observed in all three modalities with a maximum coincident with pulse onset. Critically, this signal was present during N2 (when a decision was being formed) but absent during N1 (when perceptual information was encoded, but no decision could be made). In other words, rhythmic gain control during sequential processing is a supramodal phenomenon that occurs whilst information is integrated towards a categorical decision.
\end{abstract}

\title{
Highlights
}

- Deconvolution of ongoing EEG signals during sequential numerosity integration

- Endogenous oscillatory phase $\sim 3 \mathrm{~Hz}$ predictive of subsequent numerosity judgment

- Supramodal rhythmic gating of visual, auditory, and somatosensory inputs

- Rhythmic gain control during decision-formation, but not during perceptual encoding

Keywords: decision-making, delta, EEG, oscillations, P300

Abbreviations: IR (impulse response), CPS (choice-predictive signal) 


\section{Introduction}

The electrical activity produced by the central nervous system tends to exhibit oscillatory dynamics, both at the level of local microcircuits and across large-scale cortical networks. A longstanding controversy concerns the role of neural oscillations in shaping the computations that underlie behaviour. One view holds that electrical oscillations are epiphenomena of the biophysical mechanisms underlying neural circuits, and play no causal role in information processing or behaviour (Shadlen and Movshon, 1999). An alternative account proposes that the phase, amplitude and frequency of neural oscillations actively encode information relevant to a current task (Buzsáki and Draguhn, 2004). One emerging theory suggests that during perception, neural oscillations might act to structure information processing across time, by periodically gating neural excitability and thereby providing temporal windows of privileged information processing and transfer (Schroeder and Lakatos, 2009; VanRullen et al., 2011). For example, in the sensory cortices of macaque monkeys, the gain of single-neuron responses is heightened when they fall in phase with oscillations entrained by rhythmic stimulation (Lakatos et al., 2007, 2008). Consistently, rhythmic entrainment of cortical oscillations was found to modulate human observers' performance in various perceptual tasks (e.g., Cravo et al., 2013; Henry and Obleser, 2012; Ng et al., 2012; Stefanics et al., 2010).

Evidence is mounting that psychophysical performance also depends on the phase of spontaneous, endogenously ongoing brain rhythms. Visual, auditory, or tactile stimuli are more likely to be detected or discriminated when they fall in the preferred phase of ongoing cortical oscillations near the alpha-frequency range (Ai and Ro, 2014; Busch et al., 2009; Hanslmayr et al., 2013; Mathewson et al., 2009; Strauß et al., 2015). Complementing these findings, endogenous rhythmic fluctuations at a lower frequency (delta, $\sim 1-3 \mathrm{~Hz}$ ) were recently reported during sequential accumulation of multiple inputs over time (Wyart et al., 2012). In that study, observers integrated the information provided by successive visual events occurring in rapid sequence to make a category judgment. Those events that fell in the preferred phase of an endogenous delta oscillation were found to be more influential in determining choices, as if momentary inputs were rhythmically gated during entry to a cumulative decision variable. Low-frequency 
oscillatory gating of sequential information may contribute to attentional selection and temporal anticipation (Cravo et al., 2013; Stefanics et al., 2010), and may ultimately provide periodic refractory periods that guard against catastrophic interference and information overload in neural processing (Marois and Ivanoff, 2005; Sergent et al., 2005).

However, although support for this view is growing, two factors limit the conclusions that can be drawn from existing studies. Firstly, slow endogenous fluctuations in sequential processing have thus far been studied mostly in the visual domain (Wyart et al., 2012, 2015; but see e.g., Giraud and Poeppel, 2012 for discussion of low-frequency oscillations in speech processing). It is thus unclear whether phase-dependent gain control of to-beaccumulated sensory inputs is a ubiquitous, supramodal phenomenon, or one that depends on the modality of sensory input. Secondly, the processing stage at which gating occurs remains unclear. Signals could be gated at an early processing stage, during sequential encoding, or later, during conversion to a decision signal, and extant studies have failed to distinguish between these possibilities.

Here, we addressed these two questions directly. To do this, we capitalised on data from a previously published experiment in which human observers compared the approximate number of pulses occurring in two successive intervals (N1 and N2; see Spitzer et al., 2014). In separate conditions, pulses were administered in the visual (blinks) auditory (beeps), and somatosensory (electrical pulses) domains. Here, we used convolution modelling of scalp electroencephalographic (EEG) data to estimate how the weight (or choice-predictiveness) associated with each pulse varied over time. We observed evidence for a rhythmic, phase-dependent gain control in centro-parietal signals at $\sim 3$ $\mathrm{Hz}$ in all three modalities, suggestive of a supramodal phenomenon. Critically, this modulation accompanied decision formation in interval N2 but was entirely absent in interval N1, when participants accumulated the pulses in the reference interval for later comparison. In other words, endogenous, delta-rhythmic gain control during perceptual decisions occurs at a late processing stage, during conversion of perceptual signals into a decision variable. 


\section{Materials and methods}

2.1 Subjects. Twenty-six healthy volunteers (22-35 years; 17 female, 9 male) gave written informed consent to participate in the experiment. Each participant received a reimbursement of $\mathbf{3 0}$ Euros. Two participants ( 1 female, 1 male) were excluded from analysis due to excessive ocular and movement artifacts in the EEG data. The study was approved by the ethics commission of the Free University Berlin and was conducted in accordance with the Human Subjects Guidelines of the Declaration of Helsinki.

2.2 Stimuli, Task, and Procedure. The experimental setup was described in detail in a previous paper, which focussed on working memory (delay-period) activity in the same data set (Spitzer et al., 2014). All stimuli were presented bilaterally at comfortable suprathreshold intensity levels. Visual pulses $(50 \mathrm{~ms})$ were delivered by white light-emitting diodes mounted to the left and the right of fixation on a TFT screen in front of the subject. Acoustic pulses were $1 \mathrm{kHz}$ sine tones of $50 \mathrm{~ms}$ duration (including $2.5 \mathrm{~ms}$ fade-in/out to prevent clicking artifacts), delivered by desktop loudspeakers. On somatosensory trials, square-wave electric median nerve stimulation $(200 \mu \mathrm{s})$ was delivered via pairs of adhesive electrodes attached to both wrists. Pulse sequences (N1/N2) were presented within $2 \mathrm{~s}$ integration intervals, the on- and offsets of which were signaled by a color change of the visual fixation cross (see Figure 1A). Each pulse was randomly allocated with equal probability to one of 13 equidistant positions between 100 and $1900 \mathrm{~ms}$, permitting a maximum pairwise repetition rate of $6.67 \mathrm{~Hz}$. The positions of pulses $/$ no pulses in each interval were randomly shuffled afresh on each trial in the running experiment, with no restrictions on the temporal structure of the resulting sequences. Prior to each block of trials, a visual cue ("seeing", "hearing", or "feeling") indicated the modality of the forthcoming trials. On each trial, a standard sequence (N1) was first presented (38 pulses, randomly varied), followed by a 3s delay. After the delay, the comparison sequence (N2) was presented, which always contained the number of pulses in N1 \pm 1 pulse, but with an independently randomized temporal structure. 
Subjects were instructed to respond only after N2 interval offset (cf. color change of the fixation cross, Figure $1 \mathrm{~A}$, right) by pressing a right foot pedal to indicate that N2 was larger than $\mathrm{N} 1$, and pressing a left pedal otherwise. Using pedal (rather than manual) responses averted upper limb movements that could have interfered with the somatosensory stimulation setup. On average, responses were given 916 ms after N2 interval offset. After each trial, full informative visual feedback was provided, by two "+" (correct) or "-" (incorrect) signs flanking the fixation cross for 200 ms. Each subject completed 6 sessions each comprising 6 blocks ( 2 of each modality, in counterbalanced, pseudo-random order) of 12 trials, yielding a total of 144 trials per modality. Control analysis verified that task performance levels remained stable over the course of the experiment $\left[F_{(5,115)}<1\right.$; ANOVA across task sessions $]$.

2.3 Behavioral analysis. To estimate how participants weighted the N1- and N2-intervals into subsequent choice, we used a psychophysical reverse correlation technique (Neri et al., 1999; Kiani et al., 2008). Specifically, we examined for each time bin $t$ of the two stimulation intervals the relative excess rate of pulses on trials subsequently judged "N2>N1", as compared to "N2<N1" (for a similar approach, see e.g., Raposo et al., 2012).

Excess rates were computed as

$$
\text { excess rate }_{t}=\frac{\sum_{i} u_{t, i}(" \mathrm{~N} 2>\mathrm{N} 1 ")-\sum_{i} u_{t, i}(" \mathrm{~N} 2<\mathrm{N} 1 ")}{\sum_{i} u_{t, i}(" \mathrm{~N} 2<\mathrm{N} 1 ")}
$$

where $\sum_{i} u_{t, i}$ is the sum of pulse units $u_{t, i}$ over trials $i$. Pulse units $u_{t, i}$ were defined for any given trial $i$ according to

$$
u_{t, i}=\frac{1}{N 1_{i}+N 2_{i}}
$$

for each time bin where a pulse occurred, and zeroed for each time bin where no pulse occurred. The trial-specific normalization of $u$ with respect to overall pulse count [equation (2)] ensured that the excess rates reflected the influence of information in the subsequent relative comparison between the two intervals, as was demanded in the behavioral task, rather than judgment of each interval in isolation. In the absence of 
response bias, the excess rate at time bin $t$ is expected to be zero if choices were uncorrelated with the physical input at that time (i.e., if the objective information at time $t$ had been ignored in the subjective N2-N1 comparison). To the extent that choices were correlated with the input at time $t$ (i.e., the information had weight in the decision), the excess rate will differ from zero. In our two-interval task, excess rates according to (1) will reflect decision weighting within the N2- and N1 intervals with opposite signs (since stronger weighting of N2 pulses favours "N2>N1"choices, whereas stronger weighting of N1 pulses favours the opposite choice). Thus, for comparative analysis and interpretation (Figure 1B-C), we infer decision weighting $(w)$ of the N2-interval from excess rates directly [equation (3); Figure $1 \mathrm{~B} / \mathrm{C}$, right], and the weighting of the N1-interval from excess rates with a flipped sign [equation (4); Figure 1B/C, left].

$$
\begin{gathered}
w_{t(N 2)}=\text { excess rate }_{t(N 2)} \\
w_{t(N 1)}=- \text { excess rate }_{t(N 1)}
\end{gathered}
$$

2.4 EEG recording and analysis. EEG was recorded from 64 active electrodes (BioSemi ActiveTwo, Amsterdam, Netherlands) configured according to the extended 10-20 system. Ocular activity was registered via two pairs of additional electrodes placed in standard bipolar montages (vertical and horizontal) around the eyes. Signals were digitized at $2048 \mathrm{~Hz}$, high- and low-pass filtered $\left(1-40 \mathrm{~Hz}, 5^{\text {th }}\right.$ order zero-phase Butterworth), down-sampled to $256 \mathrm{~Hz}$, and referenced to common average. The EEG was corrected for eye blinks using adaptive spatial filtering (Ille et al., 2002) and epoched $(-200 \ldots 2700 \mathrm{~ms}$ relative to interval cue onset). Remaining artifacts were rejected by excluding epochs containing amplitudes $>80 \mu \mathrm{V}$ from analysis. All analysis was performed using SPM for MEG/EEG (Wellcome Department of Cognitive Neurology, London, UK: www.fil.ion.ucl.ac.uk/spm/) and custom MATLAB code (The Mathworks, Inc., Natick, USA).

2.4.1 Impulse response modeling. For convolution analysis of EEG activity on the singlepulse level, the artifact-free epochs of interest (N1/N2) were re-concatenated to form pseudo-continuous recordings. Pulse onsets were convolved with a Fourier-basis (Figure 
$2 A)$ to create a set of orthogonal predictors of the EEG response in peri-pulse time. At this stage, prior to parameter estimation, the predictor set is generic, in that it encompasses any potential response shape within its resolution (Litvak et al., 2013). We used a $12^{\text {th }}$-order orthogonal Fourier-basis spanning -100 to $600 \mathrm{~ms}$ relative to pulse onset (Figure 2A), hence covering frequencies up to $\sim 17 \mathrm{~Hz}$ with a resolution of $\sim 1.4 \mathrm{~Hz}$. These parameters were chosen based on previous work (Wyart et al., 2012) and adjusted for computational efficacy (cf. Litvak et al., 2013), but exploratory analyses with higherorder Fourier-bases (covering higher frequencies) and/or longer convolution windows (affording finer frequency resolution) yielded qualitatively identical results. The visually cued on- and offsets of the $2 \mathrm{~s}$ intervals (cf. Figure $1 \mathrm{~A}$ ) were modeled as additional regressors of no interest, in- or exclusion of which however yielded equivalent pulse-level results. The parameters of the predictor set were estimated by a general linear model (GLM) fit of the recorded data, to obtain for each pulse type a set of regression coefficients (one for each Fourier function). The Fourier-spectrum of coefficients for each pulse type was then back-transformed into the time-domain by multiplication with the Fourier-basis, yielding the EEG signal's impulse response (IR), expressed as a series of $\beta$-coefficients in time (e.g., Figure 2B). Hereafter, the IRs were processed like conventional eventrelated potentials.

Choice predictive signals (CPS) were assessed by contrasting the pulse-level IRS between subsequent "N2>N1" and "N2<N1" judgments. For comparability between intervals (N1/N2), analogous to 2.3 Behavioral analysis above, the CPS for N1 pulses is expressed with a flipped sign (-CPS), i.e. with reversed polarity. Statistical analyses were implemented as conventional t-tests and factorial analyses on the group level, using Gaussian smoothing and Random Field Theory as implemented in SPM (Worsley et al., 1996; Kilner et al., 2005; Litvak et al., 2011) to control for family-wise errors (FWE) in full time- and channel space, at a peak-level threshold of $p=0.05$, FWEcorrected. Unless noted otherwise, CPS results are displayed for a representative set of centro-parietal channels $(\mathrm{C} 3, \mathrm{C} 1, \mathrm{C} 2, \mathrm{CP} 2, \mathrm{CP} 4)$ identified as significant in the initial overall CPS analyses (Figure 3A-B).

2.4.2 Single-sine convolution analysis. Frequency- and phase parameters were estimated by a least-squares sinusoidal fit of the mean CPS obtained from generic 
convolution modeling of the pulses in the N2 interval (Figure 3B). A single sine function with these parameters, unit amplitude, and 2 cycles length was then used to replace the generic Fourier-basis in the above convolution model (cf. Figure 4A vs Figure 2A). For cross-modal statistical testing (Figure 4B), for each modality, the phase- and frequency parameters of the single basis function were derived from a sinusoidal fit of the average N2-CPS in the two remaining modalities, respectively. The best-fitting sinusoids varied only little in $\delta$-frequency $(2.7-2.9 \mathrm{~Hz}$ ) and phase (zero-offsets between -100 and $-82 \mathrm{~ms}$ ). Of note, neither of these frequencies matched any of the pairwise stimulus repetition rates permitted by sequence design $(6.7 \mathrm{~Hz}$ and/or its subharmonics). For the subsequent analyses (Figures $4 \mathrm{C}-5 \mathrm{C}$ ), the sine parameters from fitting the overall mean CPS in the N2-interval were used (see Figure 3B, dashed grey, $2.8 \mathrm{~Hz}, 90 \mathrm{~ms}$ offset).

2.4.3 Power-regime analysis. Instantaneous oscillatory power was estimated using a Hilbert transform with center frequencies of 2.8, 6.7 and $11 \mathrm{~Hz}$ (see Results). In separate convolution GLMs (one for each center frequency), pulses onsets were median-split according to whether they occurred during a period of high or low power (relative to the median of pulses associated with the same subsequent choice), and were modeled by separate regressors in the GLM design matrix. Within each power regime, CPS analysis proceeded as outlined above.

\section{RESULTS}

Human participants $(n=24)$ experienced trains of stimulus pulses delivered irregularly within successive $2 \mathrm{~s}$ intervals (Figure $1 \mathrm{~A}$ ). On every trial, participants indicated with a pedal press whether the number of pulses in the second sequence (N2; 2-9 pulses) was higher ("N2>N1") or lower ("N2<N1") than the number of pulses in the first sequence (N1; 3-8 pulses). N2 was always N1 \pm 1 (randomly varied), such that participants could only perform the task after both sequences (N1 and N2) had been presented. On different blocks of trials, the pulses were either light blinks, tone beeps, or brief electrical stimulation of the wrists (Spitzer et al., 2014); see Materials and Methods). 
3.1 Behavioral Performance. Conventional analyses of the overall behavioral performance levels in the experiment are reported in Spitzer et al. (2014). Briefly, participants showed superior N1-N2 discrimination in the auditory (86.5\% correct) compared to the visual (78.1\%) and somatosensory (77.6\%) conditions, and a general performance decrease for higher N1/N2 numerosities, as expected by Weber's law (for details, see Spitzer et al., 2014). Here, however we asked a different question: how strongly are choices influenced by the stimulus information in each of the two intervals? To address this, we used psychophysical reverse correlation to estimate decision weight $(w)$, at each time point within the N1- and N2-intervals, respectively, with which a pulse contributed to the choice of "N2>N1" or "N2<N1" (Figure 1B). We grouped the 13 time bins in either interval into early $(<500 \mathrm{~ms})$, middle $(500-1500 \mathrm{~ms})$, and late $(>1500 \mathrm{~ms})$ windows (Figure $1 \mathrm{C}$ ), for statistical analysis in a 3-way ANOVA with the factors modality (visual, auditory, somatosensory), interval (N1,N2), and time window (early, middle, late).

The ANOVA showed a main effect of modality $(F(2,46)=5.22, p=0.009)$, mirroring the above-reported performance levels, but no main effect of interval $\left(F_{(1,23)}<1\right)$, and no significant interaction between these two factors (modality $x$ interval; $F_{(2,46)}=2.65, p=0.08$ ). Next, we examined whether choices might have been differentially driven by information arriving early or late in the stimulation intervals (so-called primacy- or recency-effects; e.g., Tsetsos et al., 2012; Cheadle et al., 2014). There was a significant main effect of time window $\left(F_{(2,46)}=7.89, p<0.001\right)$, indicating the presence of primacy/recency effects, as well as a significant time window $x$ interval interaction $\left(F_{(2,46)}=22.69, p<0.001\right)$, with primacy predominating in the N1-interval but recency being most clearly evident in the N2-interval. Lastly, the time window $x$ modality interaction was significant $\left(F_{(4,92)}=2.66\right.$, $p=0.037$ ), with less pronounced primacy/recency-effects in the somatosensory condition, however, we failed to observe a 3-way interaction across intervals (modality $x$ interval $x$

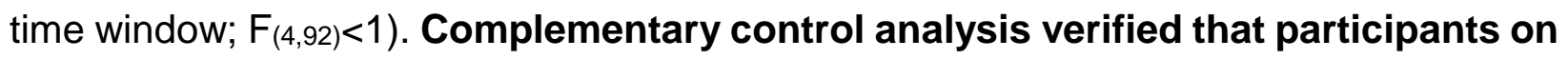
average responded "N2>N1" and "N2<N1" equally often, in all three modalities (all $p$ 's $>0.30$, paired t-tests), indicating there was no systematic response bias towards either of the two choices.

Anticipating the analyses below, which focus on modulations by endogenous, ongoing oscillations (cf. 3.3 Pulse-level choice-predictive signals), 
we verified that the stimulation occurring in our task contained no stereotypical (i.e., "phase-locked") rhythmicity throughout the N1/N2-intervals, depending on choice. To this end, we assayed the frequency spectra of the excess rates $w$ in each subject by fast Fourier transform (FFT; cf. Figure 1B, insets). The spectra disclosed no prominent peak frequencies (note upper frequency limit by sequence design, cf. 2.2 Stimuli, Task, and Procedure), and did not differ significantly across intervals (N1, N2) and/or modalities (all p's > 0.05; 3-way ANOVA with factors modality, interval, and frequency; see supplementary Figure S1C for analogue nullresult in EEG-spectra).

3.2 EEG results: Impulse response modeling. We measured neural activity evoked by single pulses in each modality, by examining EEG impulse responses (IRs) derived from a generic convolution model fit to the recorded data (Litvak et al., 2013; see Materials and Methods). In principle, the IRs obtained by this method are equivalent to event-related potentials (ERPs) obtained by traditional trial-based averaging. However, convolution modeling accounts for response overlap that may occur where stimulation is rapid and irregularly spaced (see Figure 2A), disclosing the shape of the peri-stimulus EEG response as if each pulse had been presented in isolation (Litvak et al., 2013). Figure 2B illustrates the grand mean IRs evoked by visual, auditory, and somatosensory pulses, respectively, at representative fronto-central channels. As expected on basis of the general EEG literature (Niedermeyer and Silva, 2005), the pulse-evoked responses showed large and complex differences between modalities. A $3 \times 2$ factorial analysis with the factors modality (visual, auditory, somatosensory) and pulse sequence (N1, N2) confirmed significant differences between modalities in all recording channels, throughout most of post-pulse time (20-490 ms, all time points $p<0.05$, FWE). Also illustrated in Figure 2B (right) are the characteristic posterior (visual), fronto-central (auditory), and post-central (somatosensory) maxima of early IR-deflections, corresponding to typical sensory ERP components in the respective modality (visual P100, auditory N100, and somatosensory P60). Differences between N1- and N2 pulses were evident at central channels in the proximity of $\mathrm{Cz}$, showing a more positive-going IR for 
N2-pulses (260-360ms; $p<0.05, F W E)$, with no significant interaction with factor modality (all channels and time points $p>0.05$, FWE).

3.3 Pulse-level choice-predictive signals. Next, turning to our main question of interest, we asked whether ongoing EEG signals at the time of a pulse predict its influence on the subsequent behavioral choice. Building on the logic of psychophysical reverse correlation above, we now measured psychophysiological interactions, i.e. how neural activity coincident with a pulse modulated its influence on the subsequent decision, over and above the physical occurrence of a pulse per se (i.e., over and above the weighting disclosed by behavioural analysis above; for a related approach, see Wyart et al., 2012). In the convolution analysis framework, we delineate such choice-predictive signals (CPS) by comparing the single-pulse IR where subjects subsequently responded "N2>N1" to that on trials where the response was "N2<N1" (i.e., CPS $=I^{2} " N 2>N 1$ " $-I R{ }^{N} N 2<N 1$ "). We first tested if overall (collapsed over modalities) significant CPS were evident in either of the two stimulation intervals (N1/N2), using group-level $t$-tests against zero (FWE-corrected for multiple comparisons across all channels and time-points, cf. 2.4.1 Impulse response modeling). The analysis revealed a prominent choice-predictive signal (with an apparent rhythmic trajectory; see below) in the N2 interval (Figure 3B): a significant positivity emerged over centro-parietal channels already at the time of pulse onset (peak at $10 \mathrm{~ms} ; \mathrm{p}<0.05, \mathrm{FWE}$ ), followed by a negativity of a similar topography around $190 \mathrm{~ms}$ $(p<0.01, F W E)$, and by another prominent positivity at $\sim 360 \mathrm{~ms} p<0.001, F W E)$. A further negativity, although below statistical threshold, was indicated around $520 \mathrm{~ms}$. Overall, the CPS during the N2 interval was well-described by an ongoing oscillation in the delta frequency band $(-2.8 \mathrm{~Hz}$; hereafter $\delta)$, spanning approximately two cycles in peri-pulse time (Figure 3B, dashed grey; sinusoidal fit of the overall CPS; cf. Figure 3D for illustration of the individual centro-parietal IRs associated with either type of subsequent choice). In other words, to the extent that the individual pulses in a N2 sequence coincided with the preferred/non-preferred phase of delta (cf. Figure 3B), N2 was over-/underestimated relative to N1. Interestingly, however, the converse was not true for N1 pulses: we observed no indication of any significant CPS in the N1 interval (Figure $3 \mathrm{~A} / \mathrm{C}$; all channels and time points $\mathrm{p}>0.18$, FWE) 
To test if any further choice-predictive activity at higher frequencies might have been overlooked in the above analysis of averaged CPS waveforms, we additionally inspected the frequency spectrum of CPS computed directly on the complex-valued Fourier coefficients obtained from generic convolution modeling (Figure 3E-F). This analysis confirmed a single significant peak in the $\delta$-band at $\sim 2.8 \mathrm{~Hz}$ (predominantly loading on the sine-component) for N2- but not for N1 pulses, and yielded no indication of any further effects at higher frequencies for either pulse type ( $p>0.05, \mathrm{FWE})$.

3.4 Choice-predictive signals in different modalities. The $\delta$-CPS during N2 resembled the rhythmic gain signals described in previous work in the visual domain (Wyart et al., 2015, 2012), but critically, here the effect was similarly evident in vision, audition, and somatosensation (Figure 3B, colored lines). For further assessment, we customized the generic convolution model (cf. Figure 2A) such that it selectively tests for a singular sinusoidal oscillation in peri-pulse time (Figure 4A; see Materials and Methods, singlesine convolution). We then asked if the CPS in each individual modality can be predicted from the $\delta$-phase and -frequency of the mean N2-CPS in the two remaining modalities, respectively. Indeed, these cross-modality tests confirmed a significant effect with very similar centro-parietal topographies in each of the three modalities in the N2 interval (Figure 4B right, t-tests against zero; each $\mathrm{p}<0.05$, FWE), whereas neither modality showed such effect in the N1 interval (Figure 4B left, all p's>0.05, FWE). A 3×2 factorial analysis with the factors modality (visual/auditory/somatosensory) and interval (N1/N2) verified a highly significant main effect of interval with a maximum over centro-parietal channels $(p<0.001, F W E)$, in the absence of a main effect of modality (all channels $p>0.20, F W E$ ) or interaction with it (all channels $p>0.50, F W E$ ). Together, these analyses establish the choice-predictive $\delta$-phase dynamics during the N2-interval as a supramodal phenomenon, occurring equivalently in the visual, auditory and somatosensory modalities. Further, they corroborate the absence of any such effect during the N1 period. Hereafter, we collapsed across modalities for maximum statistical power to study the effect in the N2 period in greater detail. 
3.5.1 Control analysis: error trials. One possibility is that the $\delta$-CPS in the N2 interval is attributable not to internal decision-related signals, but to systematic differences in the physical properties of stimulation: N2 sequences judged "N2>N1" on average contained more pulses than those judged "N2<N1" (6.31 vs. 4.71) and were more densely spaced (mean inter-pulse-time, IPT: 283 vs. $331 \mathrm{~ms}$ ). To test this possibility, we capitalised on error trials, on which the systematic differences between the subsequent choices were inverted. To deal with low trial counts, we first isolated from our subject sample the 12 poorest performing participants, who produced the greatest number of errors (on average $23.6 \%$ of trials), Critically, although these participants' incorrect choices were associated with a clear reversal of N2 numerosity ("N2>N1" vs. "N2<N1": 5.49 vs. 6.43 pulses) and IPT (349 vs. $289 \mathrm{~ms}$ ), their error trials showed a significant $\delta$-CPS of the same polarity (Figure 4C upper, p<0.05, FWE, t-tests against zero; cf. suppl. Figure S1A for generic convolution results), and with a very similar topography as the overall grand mean CPS. In other words, the ongoing $\delta$-phase during N2 predicted participants' subsequent choices regardless of the physical ground truth on a given trial. Moreover, the finding of similar CPS despite opposite differences in IPT renders it unlikely that the choice-predictive rhythmicity is epiphenomenal to contrasting IRs between more densely vs more sparsely spaced pulses.

3.5.2 Control analysis: numerosity-balanced trials. As a further control, we wished to test whether our findings were driven solely by the largest and smallest N2 numerosities, where the absolute number of pulses in N2 alone was diagnostic of the N2-N1 difference (i.e., if the N2 interval contained 2, 3, 8, or 9 pulses, cf. Materials and Methods). To balance this factor, returning to our full cohort, we also estimated the $\delta$ CPS separately for each of those subsets of trials where N2 was either 4, 5, 6, or 7 (each of which was equally often preceded by a larger or smaller N1). Indeed, the averaged $\delta$-CPS computed from these subsets reproduced a significant centro-parietal effect (Figure 4C, lower; $p<0.05$, FWE, t-test against zero). Further, in a factorial comparison of the individual N2-levels, we failed to observe any significant differences (all channels $p>0.05$; FWE), indicating that the $\delta$-CPS did not critically depend on the overall number of pulses in the N2-interval. 
3.5.3 No evidence for phase-reset of $\delta$-CPS during rapid pulse succession. Using convolution modeling, our analysis accounted for overlapping evoked responses during rapid stimulus presentation. Yet, the question arises to what extent the ongoing CPS itself might have been affected by (or depended on) the temporal proximity of previous input. Interestingly, when extending our analysis model to include effects of IPT (inter-pulsetime) on the individual pulse level, we found the $\delta$-CPS to be not significantly affected by the distance/proximity of preceding pulses (suppl. Figure S1B). This finding suggests that the phase of the ongoing $\delta$-CPS was not substantially "reset" (and/or driven) by preceding pulses. In a related control analysis, we examined analogous to the FFT of excess rates (cf. 3.1 Behavioral Performance), whether the centro-parietal EEG signal might have been stereotypically phase-locked (or "entrained") to N2-interval time. However, the FFT spectrum of conventionally averaged EEG epochs showed no prominent peak at the CPS-frequency, and the signal-portion in the $\delta$-band did not differ between the N2- and N1-intervals (see suppl. Figure S1C for details). Together, we failed to find factors that might relate the $\delta$-CPS to any physical/extrinsic aspects of the task, in line with an explanation of the effect in terms of an endogenously driven fluctuation.

[link inline supplementary Figure S1 about here]

3.6 Time course of the CPS during interval N2. The finding of a supramodal $\delta$-CPS in the $\mathrm{N} 2$ - but not the N1 interval supports the prediction that rhythmic sampling modulates the gain of momentary input only when decisions are being formed. A further prediction arising from this hypothesis is that the oscillatory modulation will build up gradually throughout N2 accumulation, as the number of pulses approaches the trial-specific reference encoded from sequence $\mathrm{N} 1$. We tested this prediction by estimating the centroparietal $\delta$-CPS separately for each of the individual N2 pulses in a trial, sorted by their momentary numerical distance from N1 (Figure 4D). Indeed, consistent with rhythmic encoding relative to the current decisional standard, the $\delta$-CPS increased gradually with decreasing numerical distance to $N 1$ (linear trend $p<0.005$ ). Therein, the $\delta$-signal was significantly choice-predictive (non-zero) already well before the N1 count was reached 
(-3; $p<0.05$, t-test, corrected for multiple comparisons), and increased to its maximum for the putatively decisive (or confirmatory) pulses that exceeded N1 $(+1)$. By randomness of the sequences, these latter pulses, if present, occurred mostly late in the N2 interval ( $\geq 1750 \mathrm{~ms}$ in $78 \%$ of cases). Hence, for comparability with the trajectory of behavioral decision-weighting (cf. Figure 1B), we examined the $\delta$-CPS also as a function of the pulses' time of occurrence within the N2-interval (Figure 4E). Unlike the behavioral recency-effect (cf. Figure 1B, upper right) the $\delta$-CPS built up gradually throughout N2interval time (linear trend $p<0.001$ ), and was significantly non-zero already at $1000 \mathrm{~ms}$ $(p<0.05$, corrected), before reaching its maximum at the end of the interval. Analogous analysis of the $\delta$-CPS in the $\mathrm{N} 1$ interval (Figure 4E, unsaturated color) reiterated the absence of such effect at any time during encoding of the trial-specific reference information (all p's>0.05). Together, the emergence of the pulse-level $\delta$-CPS was dissociable from the time-course of behavioural decision weighting (cf. Figure 1B), both between and within intervals. Participants' choices effectively encoded stimulus information from both $\mathrm{N} 1$ and N2 (and therein, showed brief primacy- and recency effects), whereas the rhythmic gating of momentary input evolved gradually (and only) during active comparison processing, while a decision was being formed.

3.7 Enhanced $\delta$-CPS during periods of high $\delta$-power. On theoretical grounds, phasedependent effects on cognitive processing can be expected to be enhanced when neural oscillations circulate with larger amplitudes, and thus have a greater physiological impact on momentary processing (Mathewson et al., 2011; Klimesch, 2012; see also Haegens et al., 2011). We here tested this prediction by extracting from each participant's recording the instantaneous centro-parietal $\delta$-power, and median-splitting the individual N2 pulses according to whether they occurred in a regime of high- or low power. Indeed, in the high $\delta$-power regime, the CPS was significantly enhanced (Figure 5A, $p<0.05$, FWE), indicating a greater behavioral impact of ongoing $\delta$-phase when its presumed neuromodulatory effect was stronger. Of note, pulse-level $\delta$-power per se did not differ between subsequent choices ( $>0.05$, paired t-test). For additional control, we performed 
analogous analyses also for power regimes at $\sim 6.7 \mathrm{~Hz}$ (i.e., the maximum stimulus repetition rate, cf. Materials and Methods) and at $\sim 11 \mathrm{~Hz}$ (i.e. alpha-power, which was previously found to be sensitive to attentional factors in this task, cf. Spitzer et al., 2014), neither of which however was associated with a difference in $\delta$-CPS (Figure 5B and C; both $p>0.05, F W E)$. These latter results render it less likely that the phase-power interaction within the $\delta$-band (cf. Figure 5A) might have been attributable to unspecific factors (like attentional fluctuations, or variability in overall signal-to-noise ratio). Together, the pattern of findings further corroborates the CPS as an effect of the phase (in interaction with the power) of ongoing delta-oscillations.

\section{DISCUSSION}

We recorded scalp EEG activity whilst healthy human participants compared the number of pulses in two consecutive intervals ( $\mathrm{N} 1$ and N2). We calculated how the ongoing activity that accompanied each pulse predicted the choice made by the participant (N1 > N2 or N2 > N1). Behaviourally, the information occurring in both intervals N1 and N2 contributed to choices. However, EEG signals that positively predicted choices were found only in the second epoch (N2). Consistent with recent reports, this choice-predictive activity fluctuated slowly, at approximately $3 \mathrm{~Hz}$, with a prominent peak that was contiguous with the arrival of the pulse. In other words, we replicate recent findings showing that stimuli falling in the preferred phase of ongoing oscillatory EEG activity are more likely to be detected or discriminated, or to carry more weight in a later decision (e.g., Busch et al., 2009; HansImayr et al., 2013; Strauß et al., 2015; Wyart et al., 2012). This lends credence to the view that neural oscillations are not epiphenomena, but contribute actively to perception and cognition.

The work described here builds on and advances this past research in two major respects. Firstly, unlike the established supramodal entrainment of primate sensory areas to rhythmic stimulation (Lakatos et al., 2009, e.g., 2007), behavioral effects of the phase of endogenous oscillations have previously mostly been studied in individual sensory 
modalities (Busch et al., 2009; Mathewson et al., 2009; Samaha et al., 2015); auditory: (Strauß et al., 2015) tactile: (Ai and Ro, 2014; cf. Monto et al., 2008). By contrast, we systematically compared the processing of pulses in three modalities: visual (blinks), auditory (beeps), and somatosensory (electrical pulses). Behaviourally, there were differences in discrimination performance between the modalities: participants' discrimination accuracy was higher for auditory trials, and the individual modality conditions showed different patterns of primacy/recency (the origins of which are not yet fully clear, see also e.g., Hubert-Wallander and Boynton, 2015) within the stimulation periods. Moreover, the EEG response evoked by pulses in each of the three modalities was strikingly different. Remarkably however, all three conditions showed a highly consistent, choice-predictive neural signal that fluctuated at approximately $3 \mathrm{~Hz}$, with significant peaks both coincident with pulse onset ( $\sim \mathrm{ms})$ and some $350 \mathrm{~ms}$ later. Indeed, using a single-sine fitting technique, the best-fitting parameters (phase and frequency) from either modality could be cross-fit to significantly predict the other modalities, and analysis of variance showed no reliable difference in effects across modalities. In other words, the choice-predictive ongoing oscillatory activity is a "supramodal" signal, i.e. one that is general across sensory domains.

Complementary to the frequently reported behavioral effects of pre-stimulus phase at $\sim 7$ $12 \mathrm{~Hz}$ in single-stimulus tasks (Ai and Ro, 2014; e.g., Busch et al., 2009; Hanslmayr et al., 2013; Mathewson et al., 2009; Strauß et al., 2015), our results highlight relatively slower phasic fluctuations during accumulative integration of sequential inputs over time (Wyart et al., 2012). Whilst non-invasive methods permit only cautious inferences about functional localisation, the topography and timing of the choice-predictive signals invite a comparison with classic late positive EEG responses of the P3-family (Sutton et al., 1965; Kelly and O'Connell, 2014). Indeed, an emerging view argues that late positive potentials recorded over central and parietal regions index a slow buildup of supramodal decisionrelated information following stimulus onset, termed the "centroparietal positive potential" or CPP. The precise relationship between the P300, the CPP, and the oscillatory phenomena reported by Wyart et al. $(2012,2015)$ and here remains unclear, but it is likely that they index overlapping neural signals that scale with the gain of information during 
decision-making (Kelly and O'Connell, 2014; cf. Philiastides et al., 2014). Our findings seem to mirror these responses' implication in widespread modulations of cortical processing gain (potentially via neuromodulatory dynamics of the locus coeruleusnorepinephrine system; Nieuwenhuis et al., 2005), as well as their association with psychological refractoriness (Sergent et al., 2005). In synergy, throughout sequential evidence processing, these factors might create a milieu of endogenous rhythmic entrainment, rendering P3-related signals potentially sufficient as a parsimonious electrophysiological account for the emergence of delta-periodic fluctuations. Consistently, with simple pulsed inputs, we found a somewhat faster rhythm $(\sim 3 \mathrm{~Hz})$ than was reported during demanding visuospatial categorization ( $\sim 2 \mathrm{~Hz}$; Wyart et al., 2012$)$, as is expected if the latency and duration of a P3-related refractoriness varied with the difficulty of sample evaluation (McCarthy and Donchin, 1981).

Secondly, previous studies have failed to establish whether rhythmic modulation of information during perceptual decision-making occurs early, during perceptual encoding of task-relevant information, or later, during conversion of perceptual signals into a cumulative decision variable. Initially, the argument was made that rhythmic modulation reflects the opening and closing of the "doors of perception", consistent with the primarily posterior locus of the effect (VanRullen and Koch, 2003) and with invasive recordings in the monkey that highlighted the primary sensory cortices as candidate substrates for oscillatory gain control (Schroeder and Lakatos, 2009). However, accruing findings of phase-dependent psychophysical performance in humans show central scalp topographies, unlike what might be expected from early sensory effects (e.g., Busch et al., 2009; Busch and VanRullen, 2010; Drewes and VanRullen, 2011). Some recent evidence points to a potential locus of rhythmic gating in parietal areas (HansImayr et al., 2013; see also Wyart et al., 2012; Mathewson et al., 2009), where incoming perceptual signals are known to be gradually transformed into a decision value according to the reference frame(s) of the task at hand (e.g., Bennur and Gold, 2011). Critically, in the single-stimulus detection- or discrimination tasks used in the majority of previous studies, perceptual- and decision information tend to be in a common frame of reference, rendering it difficult to distinguish at which level 
of processing the rhythmicity effectively occurred (for related discussion, see Strauß et al., 2015). Indeed, during visual multi-sample categorization, using modelbased analysis, one recent study (Wyart et al., 2012) provided evidence for rhythmic (1$3 \mathrm{~Hz}$ ) modulatory gain control at the level of the decision, but not at early perceptual stages.

Here, we capitalised on the design of a previously published experiment that was wellsuited to arbitrate between these possibilities. In our two-interval choice task, there is a fundamental asymmetry between the processing that occurs in intervals N1 and N2: in $\mathrm{N} 1$, the stimulus is perceptually encoded, but a decision cannot be formed, because there is no information that is predictive of whether N2 will be shorter or longer. By contrast, during N2, the stimulus is also perceptually encoded, but secondarily, a decision can gradually be formed, on the basis of the arriving information. Studies of somatosensory discrimination in the monkey have leveraged this asymmetry to dissociate perceptual and decision stages of processing (for review, see Romo and de Lafuente, 2012). Although intervals N1 and N2 were perceptually equivalent, and required the same accumulative encoding of the pulse stream, endogenous delta-band oscillations effectively up- and down-regulated the processing of momentary inputs only at interval N2, when the decision could be formed. Moreover, when the time course of this effect was measured by estimating choice-predictive signals for early and late pulses, we found that it grew over the N2 interval. In other words, the strength of rhythmic gain modulation tracked the evolution of the decision formation process. This strongly suggests that rhythmic gain modulation occurs at the decision, and not the perceptual, stage in our task. It does not, however, rule out the possibility that similar phenomena may occur in early sensory regions under different task conditions, or that such phenomena may be detectable with more sensitive measurement techniques, such as those that rely on invasive recordings.

Moreover, previous studies that have reported rhythmic gain modulation by intrinsic deltaband signals have used tasks in which stimuli occurred in a predictable train, e.g., at $\sim 2 \mathrm{~Hz}$ (Lakatos et al., 2008), 3Hz (Wyart et al., 2015), or $4 \mathrm{~Hz}$ (Wyart et al., 2012). These studies alone thus leave open the possibility that the rhythmic effects observed depend on the 
temporal regularity and predictability of the stimulation train. Here, however, we report a similar phenomenon in the context of pulse sequences that occurred unpredictably and irregularly. This, and the absence of rhythmic fluctuations during sequential encoding per se, demonstrates that rhythmic gain control is not an artefact of rhythmic stimulation, either at a comparable frequency or a subharmonic of that frequency. Of note, however, while endogenous rhythmic gain control has been reported in the (high) alpha (Drewes and VanRullen, 2011; e.g., Mathewson et al., 2009), low alpha (Busch et al., 2009; Busch and VanRullen, 2010; Hanslmayr et al., 2013), theta (Dugué et al., 2015), and delta bands (Wyart et al., 2015, 2012), the factors that determine the dominant modulatory frequency remain unknown. One possibility is that the frequency of the endogenous modulatory oscillation increases or decreases to match the demand of the task. For example, detecting a small luminance increment depends on the phase of lower alpha-band activity $(\sim 8 \mathrm{~Hz})$; counting a train of pulses depends on high delta-band activity $(\sim 3 \mathrm{~Hz})$, whereas integrating a complex, nonlinear categorical variable depends on the phase of $\sim 2 \mathrm{~Hz}$ activity. However, this is a speculation that awaits empirical testing.

The demonstration of rhythmic gain control during decision-making places a new constraint on computational models of perceptual choice. The field is dominated by models in which information accumulates linearly to a decision threshold (Gold and Shadlen, 2007; Ratcliff and Smith, 2004), but the role of adaptation, attention and/or resource allocation during information integration has been less well explored. Our data offer as-yet-untested predictions about the factors that should shape the buildup of firing rates in frontal and parietal regions during discrimination of psychophysical stimuli, such as a random dot kinetogram (Beck et al., 2008; Kim and Shadlen, 1999; Shadlen and Newsome, 2001), and in particular that there should be a relationship between firing rates and ongoing activity in the local field potential that predicts choices in such situations. We hope that other researchers with access to invasive recording data will be inspired to test these predictions in future work. 
Acknowledgements: This work was supported by a grant from the German Research Foundation to BS (DFG SP 1510/1-1). We wish to thank Vladimir Litvak and Guillaume Flandin for technical support, Simon Hanslmayr, Valentin Wyart, and two anonymous reviewers for helpful suggestions, and Sebastian Fleck for data acquisition assistance.

References

Ai, L., Ro, T., 2014. The phase of prestimulus alpha oscillations affects tactile perception. J. Neurophysiol. 111, 1300-1307. doi:10.1152/jn.00125.2013

Beck, J.M., Ma, W.J., Kiani, R., Hanks, T., Churchland, A.K., Roitman, J., Shadlen, M.N., Latham, P.E., Pouget, A., 2008. Probabilistic Population Codes for 
Bayesian Decision Making. Neuron 60, 1142-1152.

doi:10.1016/j.neuron.2008.09.021

Bennur, S., Gold, J.I., 2011. Distinct representations of a perceptual decision and the associated oculomotor plan in the monkey lateral intraparietal area. J. Neurosci. 31, 913-921. doi:10.1523/JNEUROSCI.4417-10.2011

Busch, N.A., Dubois, J., VanRullen, R., 2009. The Phase of Ongoing EEG Oscillations Predicts Visual Perception. J. Neurosci. 29, 7869-7876. doi:10.1523/JNEUROSCI.0113-09.2009

Busch, N.A., VanRullen, R., 2010. Spontaneous EEG oscillations reveal periodic sampling of visual attention. PNAS 107, 16048-16053. doi:10.1073/pnas.1004801107

Buzsáki, G., Draguhn, A., 2004. Neuronal oscillations in cortical networks. Science 304, 1926-1929. doi:10.1126/science.1099745

Cheadle, S., Wyart, V., Tsetsos, K., Myers, N., de Gardelle, V., Herce Castañón, S., Summerfield, C., 2014. Adaptive gain control during human perceptual choice. Neuron 81, 1429-1441. doi:10.1016/j.neuron.2014.01.020

Cravo, A.M., Rohenkohl, G., Wyart, V., Nobre, A.C., 2013. Temporal expectation enhances contrast sensitivity by phase entrainment of low-frequency oscillations in visual cortex. J. Neurosci. 33, 4002-4010. doi:10.1523/JNEUROSCI.467512.2013

Drewes, J., VanRullen, R., 2011. This is the rhythm of your eyes: the phase of ongoing electroencephalogram oscillations modulates saccadic reaction time. J. Neurosci. 31, 4698-4708. doi:10.1523/JNEUROSCI.4795-10.2011

Dugué, L., Marque, P., VanRullen, R., 2015. Theta oscillations modulate attentional search performance periodically. J Cogn Neurosci 27, 945-958. doi:10.1162/jocn_a_00755

Giraud, A.-L., Poeppel, D., 2012. Cortical oscillations and speech processing: emerging computational principles and operations. Nat Neurosci 15, 511-517. doi:10.1038/nn.3063

Gold, J.I., Shadlen, M.N., 2007. The neural basis of decision making. Annu Rev Neurosci 30, 535-74.

Haegens, S., Nácher, V., Luna, R., Romo, R., Jensen, O., 2011. a-Oscillations in the monkey sensorimotor network influence discrimination performance by rhythmical inhibition of neuronal spiking. Proc. Natl. Acad. Sci. U.S.A. 108, 19377-19382. doi:10.1073/pnas.1117190108

Hanslmayr, S., Volberg, G., Wimber, M., Dalal, S.S., Greenlee, M.W., 2013. Prestimulus Oscillatory Phase at $7 \mathrm{~Hz}$ Gates Cortical Information Flow and Visual Perception. Current Biology 23, 2273-2278. doi:10.1016/j.cub.2013.09.020

Henry, M.J., Obleser, J., 2012. Frequency modulation entrains slow neural oscillations and optimizes human listening behavior. Proc. Natl. Acad. Sci. U.S.A. 109, 20095-20100. doi:10.1073/pnas.1213390109

Hubert-Wallander, B., Boynton, G.M., 2015. Not all summary statistics are made equal: Evidence from extracting summaries across time. J Vis 15, 5. doi:10.1167/15.4.5

Ille, N., Berg, P., Scherg, M., 2002. Artifact correction of the ongoing EEG using spatial filters based on artifact and brain signal topographies. J Clin Neurophysiol 19, 113-124. 
Kelly, S.P., O'Connell, R.G., 2014. The neural processes underlying perceptual decision making in humans: Recent progress and future directions. J. Physiol. Paris. doi:10.1016/j.jphysparis.2014.08.003

Kiani, R., Hanks, T.D., Shadlen, M.N., 2008. Bounded integration in parietal cortex underlies decisions even when viewing duration is dictated by the environment. J. Neurosci. 28, 3017-3029. doi:10.1523/JNEUROSCI.4761-07.2008

Kilner, J.M., Kiebel, S.J., Friston, K.J., 2005. Applications of random field theory to electrophysiology. Neurosci. Lett 374, 174-178. doi:10.1016/j.neulet.2004.10.052

Kim, J.-N., Shadlen, M.N., 1999. Neural correlates of a decision in the dorsolateral prefrontal cortex of the macaque. Nat Neurosci 2, 176-185. doi:10.1038/5739

Klimesch, W., 2012. Alpha-band oscillations, attention, and controlled access to stored information. Trends Cogn Sci 16, 606-617. doi:10.1016/j.tics.2012.10.007

Lakatos, P., Chen, C.-M., O'Connell, M.N., Mills, A., Schroeder, C.E., 2007. Neuronal Oscillations and Multisensory Interaction in Primary Auditory Cortex. Neuron 53, 279-292. doi:10.1016/j.neuron.2006.12.011

Lakatos, P., Karmos, G., Mehta, A.D., Ulbert, I., Schroeder, C.E., 2008. Entrainment of Neuronal Oscillations as a Mechanism of Attentional Selection. Science 320, 110-113. doi:10.1126/science.1154735

Lakatos, P., O'Connell, M.N., Barczak, A., Mills, A., Javitt, D.C., Schroeder, C.E., 2009. The leading sense: supramodal control of neurophysiological context by attention. Neuron 64, 419-430. doi:10.1016/j.neuron.2009.10.014

Litvak, V., Jha, A., Flandin, G., Friston, K., 2013. Convolution models for induced electromagnetic responses. Neuroimage 64, 388-398. doi:10.1016/j.neuroimage.2012.09.014

Litvak, V., Mattout, J., Kiebel, S., Phillips, C., Henson, R., Kilner, J., Barnes, G., Oostenveld, R., Daunizeau, J., Flandin, G., Penny, W., Friston, K., 2011. EEG and MEG Data Analysis in SPM8. Comput Intell Neurosci 2011. doi:10.1155/2011/852961

Marois, R., Ivanoff, J., 2005. Capacity limits of information processing in the brain. Trends Cogn. Sci. (Regul. Ed.) 9, 296-305. doi:10.1016/j.tics.2005.04.010

Mathewson, K.E., Gratton, G., Fabiani, M., Beck, D.M., Ro, T., 2009. To see or not to see: prestimulus alpha phase predicts visual awareness. J. Neurosci. 29, 27252732. doi:10.1523/JNEUROSCI.3963-08.2009

Mathewson, K.E., Lleras, A., Beck, D.M., Fabiani, M., Ro, T., Gratton, G., 2011. Pulsed out of awareness: EEG alpha oscillations represent a pulsed-inhibition of ongoing cortical processing. Front. Psychology 2, 99. doi:10.3389/fpsyg.2011.00099

McCarthy, G., Donchin, E., 1981. A metric for thought: a comparison of P300 latency and reaction time. Science 211, 77-80. doi:10.1126/science.7444452

Monto, S., Palva, S., Voipio, J., Palva, J.M., 2008. Very slow EEG fluctuations predict the dynamics of stimulus detection and oscillation amplitudes in humans. J. Neurosci. 28, 8268-8272. doi:10.1523/JNEUROSCI.1910-08.2008

Neri, P., Parker, A.J., Blakemore, C., 1999. Probing the human stereoscopic system with reverse correlation. Nature 401, 695-698. doi:10.1038/44409

$\mathrm{Ng}$, B.S.W., Schroeder, T., Kayser, C., 2012. A precluding but not ensuring role of entrained low-frequency oscillations for auditory perception. J. Neurosci. 32, 12268-12276. doi:10.1523/JNEUROSCI.1877-12.2012 
Niedermeyer, E., Silva, F.H.L. da, 2005. Electroencephalography: Basic Principles, Clinical Applications, and Related Fields. Lippincott Williams \& Wilkins.

Nieuwenhuis, S., Aston-Jones, G., Cohen, J.D., 2005. Decision making, the P3, and the locus coeruleus-norepinephrine system. Psychol Bull 131, 510-532. doi:10.1037/0033-2909.131.4.510

Philiastides, M.G., Heekeren, H.R., Sajda, P., 2014. Human Scalp Potentials Reflect a Mixture of Decision-Related Signals during Perceptual Choices. J. Neurosci. 34, 16877-16889. doi:10.1523/JNEUROSCI.3012-14.2014

Raposo, D., Sheppard, J.P., Schrater, P.R., Churchland, A.K., 2012. Multisensory Decision-Making in Rats and Humans. J. Neurosci. 32, 3726-3735. doi:10.1523/JNEUROSCI.4998-11.2012

Ratcliff, R., Smith, P.L., 2004. A comparison of sequential sampling models for twochoice reaction time. Psychol Rev 111, 333-367. doi:10.1037/0033295X.111.2.333

Romo, R., de Lafuente, V., 2012. Conversion of sensory signals into perceptual decisions. Progress in neurobiology. doi:10.1016/j.pneurobio.2012.03.007

Samaha, J., Bauer, P., Cimaroli, S., Postle, B.R., 2015. Top-down control of the phase of alpha-band oscillations as a mechanism for temporal prediction. Proc. Natl. Acad. Sci. U.S.A. 112, 8439-8444. doi:10.1073/pnas.1503686112

Schroeder, C.E., Lakatos, P., 2009. Low-frequency neuronal oscillations as instruments of sensory selection. Trends Neurosci. 32, 9-18. doi:10.1016/j.tins.2008.09.012

Sergent, C., Baillet, S., Dehaene, S., 2005. Timing of the brain events underlying access to consciousness during the attentional blink. Nat Neurosci 8, 13911400. doi:10.1038/nn1549

Shadlen, M.N., Movshon, J.A., 1999. Synchrony unbound: a critical evaluation of the temporal binding hypothesis. Neuron $24,67-77,111-125$.

Shadlen, M.N., Newsome, W.T., 2001. Neural basis of a perceptual decision in the parietal cortex (area LIP) of the rhesus monkey. J. Neurophysiol. 86, 1916-1936.

Spitzer, B., Fleck, S., Blankenburg, F., 2014. Parametric Alpha- and Beta-Band Signatures of Supramodal Numerosity Information in Human Working Memory. J. Neurosci. 34, 4293-4302. doi:10.1523/JNEUROSCI.4580-13.2014

Stefanics, G., Hangya, B., Hernádi, I., Winkler, I., Lakatos, P., Ulbert, I., 2010. Phase entrainment of human delta oscillations can mediate the effects of expectation on reaction speed. J. Neurosci. 30, 13578-13585. doi:10.1523/JNEUROSCI.070310.2010

Strauß, A., Henry, M.J., Scharinger, M., Obleser, J., 2015. Alpha Phase Determines Successful Lexical Decision in Noise. J. Neurosci. 35, 3256-3262. doi:10.1523/JNEUROSCI.3357-14.2015

Sutton, S., Braren, M., Zubin, J., John, E.R., 1965. Evoked-Potential Correlates of Stimulus Uncertainty. Science 150, 1187-1188. doi:10.1126/science.150.3700.1187

Tsetsos, K., Gao, J., McClelland, J.L., Usher, M., 2012. Using Time-Varying Evidence to Test Models of Decision Dynamics: Bounded Diffusion vs. the Leaky Competing Accumulator Model. Front Neurosci 6, 79. doi:10.3389/fnins.2012.00079 
VanRullen, R., Busch, N.A., Drewes, J., Dubois, J., 2011. Ongoing EEG Phase as a Trial-by-Trial Predictor of Perceptual and Attentional Variability. Front Psychol 2, 60. doi:10.3389/fpsyg.2011.00060

VanRullen, R., Koch, C., 2003. Is perception discrete or continuous? Trends Cogn. Sci. (Regul. Ed.) 7, 207-213.

Worsley, K.J., Marrett, S., Neelin, P., Vandal, A.C., Friston, K.J., Evans, A.C., F, 1996. A Unified Statistical Approach for Determining Significant Signals in Images of Cerebral Activation. HUMAN BRAIN MAPPING 4, 58-73.

Wyart, V., de Gardelle, V., Scholl, J., Summerfield, C., 2012. Rhythmic fluctuations in evidence accumulation during decision making in the human brain. Neuron 76 , 847-858. doi:10.1016/j.neuron.2012.09.015

Wyart, V., Myers, N.E., Summerfield, C., 2015. Neural mechanisms of human perceptual choice under focused and divided attention. J. Neurosci. 35, 34853498. doi:10.1523/JNEUROSCI.3276-14.2015 
Figure 1. $A$, Sequential two-interval numerosity comparison task. Participants were presented with random sequences of brief stimulus pulses (illustrated by purple dots) in either the visual (blinks), auditory (beeps), or somatosensory modality (electric pulses). On each trial, the number of pulses in the second interval (N2) was to be judged as higher or lower compared to the first interval (N1). B, Behavioral decision weighting (w) throughout the N1- (left) and N2 (right) intervals, derived from psychophysical reverse correlation analysis (see 2.3 Behavioral Analysis). Inset plots show mean power spectra derived from FFT-analysis of the time-courses of $w$ in each subject. $C$, Decision weighting, same as $B$, but aggregated across time points for statistical analysis (see Results, 3.1 Behavioral Performance). Error bars show standard error of the mean.

Figure 2. A, EEG impulse response modeling. Pulse onsets were convolved with a generic set of orthogonal Fourier basis functions. Each horizontal line in the greyscale image corresponds to a basis function (sine/cosine). Note area of overlap for nearby pulses (right). Regression coefficients for each basis function were obtained by a GLM-fit to the EEG recording. Back-transformation of the coefficient spectrum yields the EEG impulse response (IR) in peri-pulse time (see Materials and Methods, 2.4.1). B, left: grand mean single-pulse IRs in the three presentation modalities, for illustrative fronto-central channels. Dashed lines: N1 pulses; solid lines: N2 pulses. Marker lines on bottom indicate significant differences $(p<0.05$, FWE) between modalities (purple), and between sequences (N1/N2; grey); right: scalp topographies of modalityspecific early IR deflections (cf. light color shadings in left), corresponding to typical components of visual (top), auditory (middle), and somatosensory (bottom) evoked responses. Topographies show average over all pulses (N1 and N2) in the respective modality. Grey dots indicate the respective components' peak channels.

Figure 3. $\boldsymbol{A}-\boldsymbol{B}$, Pulse-level choice-predictive signals (CPS) were assayed as the difference in single-pulse IR between the two types of subsequent choice ("N2>N1" vs "N2<N1"). For comparability, the CPS results for the N1- ( $\boldsymbol{A}$, note inverted y-axis) and N2 intervals $(\boldsymbol{B})$ are shown for identical centro-parietal channels. Topographical statistical maps on top are shown for the overall CPS, collapsed across modalities. Dashed gray curve in $\boldsymbol{B}$ shows sinusoidal fit of the overall CPS during N2 ( $2.8 \mathrm{~Hz})$. $\boldsymbol{C}-\boldsymbol{D}$, Grand mean centro-parietal impulse response (collapsed across modalities), plotted separately for either subsequent choice. Orange marker lines on $x$-axis indicate significant differences. E-F, Overall CPS, analogous to A-B dark grey, but computed directly on the Fourier-coefficients obtained from generic convolution modelling (i.e., prior to back-projection into the time-domain, cf. Materials and Methods, 2.4.1 Impulse response modeling). 
Figure 4. $A$, For further analyses, the Fourier basis set of the generic convolution model (cf. Figure 2A) was replaced by a single sinusoidal basis function with fixed phase and frequency. $B$, Cross-modal analysis. Topographical statistical maps show for each modality the $\delta$-CPS obtained from a single-sine convolution model where the phase and frequency of the basis function (see $A$ ) were derived from the average N2-CPS in the two remaining modalities (see Figure 3B), respectively. C, Control analyses. upper, $\delta$-CPS during the N2-interval, computed from error trials only. Results are collapsed across modalities and include only the 12 lowest-performing subjects (median split) in which a sufficient number of error trials was available. lower, $\delta$-CPS for the full cohort $(n=24)$, but computed from trial subsets with balanced N2 pulse counts only (4...7; cf. Results 3.5.2). $D$, Grand mean centro-parietal $\delta$-CPS during N2, as a function of the distance of the momentary N2 pulse count from the trial-specific pulse count in N1. Error bars show standard error of the mean. Solid line shows linear fit. Inset shows average CPS waveforms obtained from generic convolution modeling, for subsets of pulses as outlined by colored marker lines in main figure. $E$, Saturated color: Same as $\mathbf{D}$, but as a function of N2 interval time. Analogous analysis of N1 pulses (light color) yielded no significant effects.

Figure 5. Enhanced rhythmic gain modulation during periods of high $\delta$-power. A, left: Grand mean N2-CPS, analogous to Figure 3B (dark grey), but computed separately for pulses that occurred during periods of high- or low $\delta$-power (median-split). Right, bar graph illustrates the average level of $\delta$-power at pulse onset under each power regime, and for each type of subsequent choice. Statistical topographical maps show $\delta$-CPS obtained from single-sine convolution modeling. $B-C$, same as $A$ right, but for power regimes defined at center frequencies of $6.7 \mathrm{~Hz}(B)$ and $11 \mathrm{~Hz}(C)$. See Results (3.8) for details.

Supplementary Figure S1. A. N2-CPS waveform at centro-parietal channels (C2, CP2, cf. Figure 4), including only the 12 lowest-performing subjects and computed separately for correct and incorrect choices. Note preserved $\delta$-phasic trajectory for incorrect trials (see also Figure 4C upper). Inset plot shows the CPS on incorrect trials computed from Fourier-coefficients. The effect peaked at the same sine component $(\sim 2.8 \mathrm{~Hz})$, with a very similar topography as the overall grand mean CPS (cf. Figure 3F). B. No evidence for phase-reset of $\delta$-CPS during rapid pulse succession. Single-sine convolution results from an extended GLM in which the individual N2-pulses were modeled separately according to whether they were immediately preceded by another pulse (upper; $150 \mathrm{~ms}$ inter-pulse-time, IPT), or by a longer period without a pulse (middle, $>300 \mathrm{~ms}$ IPT). In both contexts, a $\delta$-CPS of the same polarity was observed, with a similar centro-parietal topography. Direct comparison (bottom) showed no significant differences between these effects ( $p>0.05 ; F W E$ ). C. FFT spectrum ("evoked power") of mean centro-parietal 
EEG signals obtained from conventional epoching and averaging across trials. Epochs were time-locked (upper) to interval onset (0-2000 ms in Figure 1A) or (lower) to the $1^{\text {st }}$ pulse of each sequence. Each spectrum showed an anticipated peak around 6-7Hz, which is expected by accruing pulse-evoked responses at each of the 13 time bins (every $150 \mathrm{~ms},-6.67 \mathrm{~Hz}$; cf. Materials and Methods) when averaging across trials. The spectra showed no further prominent peaks in excess of the typical 1/f noise of EEG. Any differences between the N1- and N2- intervals were unsystematic, and confined to frequencies $\geq 4 \mathrm{~Hz}$ (cf. orange markers, $p<0.01$, uncorrected). 